\title{
5-fluorouracil induced cardiotoxicity: Review of the literature
}

\author{
Michael F. Sorrentino ${ }^{1}$, Jiwon Kim², Andrew E. Foderaro ${ }^{1}$, Alexander G. Truesdell ${ }^{2}$
}

${ }^{1}$ Department of Medicine, Warren Alpert Medical School of Brown University, Providence, Rhode Island, USA ${ }^{2}$ Division of Cardiology, Warren Alpert Medical School of Brown University, Providence, Rhode Island, USA

\begin{abstract}
5-fluorouracil (5-FU) is a key chemotherapeutic agent in the treatment of many gastrointestinal tract adenocarcinomas. Despite its proven therapeutic efficacy, 5-FU also possesses several undesired cardiac toxicities, including coronary vasospasm, coronary thrombosis, cardiomyopathy, and sudden cardiac death. This review addresses the incidence, mechanisms of action, clinical presentation, risk stratification, and management of 5-FU associated cardiotoxicity; it also highlights the importance of careful pre-administration cardiac risk stratification and close monitoring during and after drug administration. (Cardiol J 2012; 19, 5: 453-458)
\end{abstract}

Key words: 5-fluorouracil, acute coronary syndrome, cardiotoxicity

\section{Introduction}

5-flourouracil (5-FU) has been a mainstay of the chemotherapeutic treatment of gastrointestinal malignancies for many years. Unfortunately, in addition to its beneficial antitumor effects, 5-FU also possesses a number of important toxicities. Acute coronary syndrome (ACS) precipitated by the administration of 5-FU is a rare but well-established phenomenon $[1,2]$ and only one of several adverse cardiac effects related to this chemotherapeutic agent. Additional cardiotoxic effects include cardiomyopathy, vasospastic angina, coronary thrombosis and dissection, malignant arrhythmias, and sudden cardiac death [3-7]. Furthermore, following any cardiac complication, it is essential to weigh the risks of repeat drug administration against the potential for cure of the malignancy given this agent's proven efficacy. This review of the literature examines the cardiac complications of 5-FU and their impact on therapeutic decision-making process, taking into consideration epidemiology, mechanisms of toxicity, clinical presentation, risk stratification, treatment, and reintroduction of 5-FU.
Table 1. Malignancies treated by 5-fluorouracil: Labeled and unlabeled use.

\begin{tabular}{cc}
\hline Labeled use & Unlabeled use \\
\hline Colorectal & Head and neck \\
Breast & Esophageal \\
Pancreas & Cervical \\
Stomach & Renal \\
& Bladder \\
& Unknown primary \\
\hline
\end{tabular}

5 -FU is a fluoropyrimidine antimetabolite agent key to several chemotherapy regimens, particularly the treatment of gastrointestinal tract adenocarcinomas (Table 1). Combined with surgical resection, 5-FU therapy offers an impressive absolute risk reduction of 5 -year mortality of $5.8 \%$ in patients with resectable gastric cancer [8]. Unfortunately, like most chemotherapeutic agents, 5 -FU also has numerous toxic effects. The most common toxicities associated with 5-FU include diarrhea, mucositis, myelosuppression, and thrombophlebitis of pe-

Address for correspondence: Michael F. Sorrentino, MD, Department of Medicine, Warren Alpert Medical School of Brown University, 593 Eddy Street, Providence, Rhode Island, 02903, tel: 631241 4913, e-mail: msorrentino1@lifespan.org

Received: 02.07.2012

Accepted: 01.08.2012 
Table 2. Cardiac toxicities reported with 5-fluorouracil administration [3-7].

\begin{tabular}{ll}
\hline Angina & Supraventricular tachycardia \\
Myocardial infarction & Prolonged QT interval \\
Congestive heart failure & Sudden death \\
Cardiomyopathy & Cardiogenic shock \\
Myopericarditis & Coronary dissection \\
Ventricular tachycardia & \\
\hline
\end{tabular}

ripheral veins [9]. The wide range of cardiotoxicities related to 5 -FU occur less frequently but are typically more serious and include ventricular arrhythmias, cardiomyopathy with significant left ventricular systolic dysfunction, arterial vasospasm, and direct endothelial toxicity, often culminating in myocardial infarction (MI), heart failure (HF), and cardiogenic shock [3-7] (Table 2). 5-FU cardiotoxicity is, in fact, the second most common cause of chemotherapy induced cardiotoxicity, second only to anthracycline cardiotoxicity $[10,11]$.

\section{Epidemiology}

The reported incidence of cardiotoxicity related to 5 -FU ranges from $1.2 \%$ to $18 \%$ [1, 2, 12-14]. This wide variation is believed to be related to dose dependency as well as the frequency of drug administration. Tsibiribi et al. [15] evaluated 1350 patients with no prior history of cardiac disease and analyzed the number of cardiac complications. In their patient population, 16 cases of cardiac complications (1.2\%) were noted. Cardiac complications reported in this study included HF, MI and angina. A similar overall incidence has been reported from other large prospective studies with an overall range of $1.2 \%$ to $8 \%$ $[1,2,12,13]$. With shorter bolus regimens, the incidence of cardiotoxicity typically lies between $1.6 \%$ to $3 \%$ of cases [2]. With more prolonged regimens, these percentages increase to $7.6 \%$ to $18 \%$ [14]. Recently, a review of 377 cases of 5-FU associated cardiotoxicity confirmed that the majority of cases of cardiotoxicity occur in the setting of continuous infusion [6]. And while overall cardiac toxicity with 5-FU administration may vary, rates are uniformly higher in patients with underlying heart disease, including coronary artery disease $(\mathrm{CAD})$, structural heart disease, and cardiomyopathies [2, 16].

\section{Mechanisms of toxicity}

The precise etiology and pathophysiology of 5 -FU cardiotoxicity is still unknown and based pri-
Table 3. Proposed mechanisms of 5-fluorouracil induced cardiotoxicity $[12,14,16,34,35,39,41]$.

\section{Coronary artery spasm}

Autoimmune-mediated injury of the myocardium

Endothelial damage

Thrombogenic effects or thrombus formation

Direct myocardial toxicity causing necrosis

Global dysfunction

Accumulation of metabolites

marily on limited animal studies, case reports, and small clinical studies. Numerous mechanisms have been proposed (Table 3). Clinically significant coronary vasospasm causing myocardial ischemia is the most commonly suspected mechanism, occurring in $1.2-18 \%$ of cases, even in the absence of CAD [1, 4, 17-19]. Both animal and human studies demonstrate a dose dependent vasospasm that ceases with cessation of drug administration [2, 4]. Experimental evidence also supports a direct toxic effect of the 5-FU on the coronary endothelium [20] and a hypercoagulable state which together precipitate acute thrombotic events $[3,19]$.

\section{Coronary vasospasm}

The precise pathogenesis of 5-FU associated cardiotoxicity has yet to be clearly elucidated. Coronary vasospasm has historically been accepted to be the main contributor to this clinical entity. Ultrasound and angiographic studies demonstrate both coronary artery and brachial artery vasospasm following 5-FU infusion [21-23]. Prior case reports reveal disease-free coronary arteries on angiography [24]. Other studies note the efficacy of vasodilator therapy, including nitrates and calcium channel blockers, in resolving chest pain and dynamic ECG changes in the setting of 5-FU administration $[24,25]$. 5 -FU also has been shown in vitro to induce vasoconstriction of vascular smooth muscle cells via the activation of protein kinase C, which resolves with administration of protein kinase inhibitors [4]. Taken together, this data supports the theory of coronary artery vasospasm as an important contributor to 5 -FU induced cardiotoxicity.

\section{Endothelial dysfunction}

Endothelial dysfunction and thrombus formation, independent of vasoconstriction, have also been shown to serve as potential mechanisms of the cardiotoxic effects of 5-FU administration. Several animal studies examined the direct effects of 5-FU on vascular endothelial cells and noted direct en- 
dothelial damage and platelet and fibrin accumulation with increased thrombus formation on both gross examination and electron microscopic evaluation [20,26]. With exposure to 5-FU, damage to endothelial cells followed by platelet accumulation and fibrin formation was observed [26].

Kuzel et al. [27] demonstrated an increase in the quantity of fibrinopeptide $\mathrm{A}$ and a decrease in the amount of protein $\mathrm{C}$ activity in the presence of 5 -FU, which together make a blood vessel more susceptible to thrombus formation. Another study comparing the adverse effects of 5-FU and methotrexate on arterial endothelial cells showed an increased incidence of endothelial injury and subsequent thrombosis with 5-FU administration [28].

\section{Direct myocardial injury}

Direct myocardial injury has also been suggested to play a role in $5-\mathrm{FU}$ induced cardiotoxicity. De Forni et al. [12] suggested the possibility of a direct drug (or drug-metabolite) toxic action on the myocardium, particularly given evidence that there is global systolic dysfunction, which does not correspond to any individual coronary artery territory. Another animal study demonstrated different toxic effects depending on the dose and frequency of drug administration [29]. A single large dose demonstrated massive hemorrhagic MI associated with coronary spasm, while repeated lower dose infusions resulted in left ventricular hypertrophy with necrosis, thickening of arteries, and apoptosis of endothelial cells. This same study also demonstrated that 5-FU can cause a diffuse myocarditis instead of vasospasm with subendocardial sparing along with inflammation [29]. Another case report utilizing ventricular biopsy demonstrated sarcoplasmic reticulum dilatation, similar to doxorubicin-associated cardiomyopathy [30].

\section{Accumulation of toxic metabolites}

The generation of metabolites of 5-FU is also likely to serve as another important mediator of this drug's cardiotoxicity of this drug. One such downstream metabolite is alpha-fluoro-beta-alanine (FBAL). Muneoka et al. [31] showed both elevated levels of FBAL after 5-FU induced MI as well as the absence of ischemia with subsequent treatment with a derivative of 5-FU which does not metabolize to FBAL.

\section{Other mechanisms}

Several other unique mechanisms of cardiotoxicity have been proposed. A study analyzing erythyrocyte membranes suggested 5 -FU induced changes to erythyrocyte membranes causing increased fluidity and conversion to echinocytic shapes. The membrane structural alterations caused functional changes diminished the erythrocyte's ability to deliver and transport oxygen, leading in turn to ischemia and myocardial injury [32]. Another study by Karabay et al. [33] suggested a Kounis syndrome as a potential toxic etiology, with a 5-FU induced allergic insult causing coronary vasospasm, which was partially responsive to antihistamine therapy. Kounis syndrome can be vasospastic or nonvasospastic, and is caused by an allergic insult causing a release of inflammatory mediators that can rupture coronary artery plaques. It typically responds to glucocorticoids and antihistamines, along with vasodilators such as nitrites and calcium channel blockers - however, given the fact that it can be either vasospastic or not, varying results with medications (such as verapamil) are experienced.

\section{Clinical presentation}

One of the earliest papers describing 5-FU cardiotoxicity illustrated that patients with cardiotoxicity secondary to 5-FU administration present with chest pain, ST and T wave ECG changes, and supraventricular and ventricular arrhythmias [34]. Other authors have noted chest pain with dynamic ECG changes as well as ST elevation MI [12, 35]. Finally, regional left ventricular wall motion abnormalities and global systolic dysfunction have also been observed [12].

Cardiotoxicity with 5-FU infusions tends occur most commonly during the first cycle of administration [35]. The median time to symptoms is $12 \mathrm{~h}$ following initiation of the infusion with a range between $3 \mathrm{~h}$ and $18 \mathrm{~h}$ [17], although in animal studies median times to symptom-onset have been more variable and have been noted to occur as late as $48 \mathrm{~h}$ into an infusion [28]. Another study also suggests earlier onset and increased severity of symptoms with higher dose regimens or repeat drug administration [36].

\section{Angina}

Angina has been shown to be one of the most common cardiac symptoms associated with 5 -FU administration [14]. Up to $19 \%$ of the patients in a series of patients by Wacker et al. [37] experienced angina during treatment, with episodes lasting up to $12 \mathrm{~h}$ after cessation of drug infusion. In the most striking case series, nearly $90 \%$ of patients experienced angina, often with troponin release or diagnostic ECG changes following 5-FU infusion [17]. 


\section{Silent ischemia and myocardial infarction}

Isolated ST changes without anginal symptoms have also been commonly observed on continuous ambulatory ECG monitoring in patients receiving 5-FU therapy. A study by Rezkalla et al. [16] demonstrated ischemic and arrhythmic ECG changes, to include ST-segment deviation and QT-prolongation, in nearly $68 \%$ of patients receiving infusions of 5-FU. Others have noted incidences of asymptomatic diagnostic ECG changes in up to $88 \%$ of patients during 5 -FU infusion, with nearly $7 \%$ demonstrating elevated cardiac enzymes [12, 35]. Another retrospective examination of 377 case reports revealed acute $\mathrm{MI}$ in $22 \%$ of patients during 5 -FU administration [6], while a prospective study of 26 patients suggested infarction by ECG and symptom criteria in nearly $35 \%$ of patients receiving 5 -FU [1]. Coronary artery dissection causing an ACS has also been described with vasospasm-induced disruption of the endothelium cited as a possible mechanism of action [38].

\section{Arrhythmia}

Transient, asymptomatic bradycardia has been also been reported with 5-FU infusion. In a study by Talapatra et al. [39], 6 patients experienced bradycardia following treatment with continuous 5-FU infusion. Significant prolongation of QTc intervals and frequent ventricular arrhythmias have also been found on Holter monitoring associated with 5-FU infusion [37]. A review of 22 patients demonstrated significant early prolongations of the QT interval which persisted throughout the duration of chemotherapy [40] and another case series described QT prolongation with torsade de pointes in a patient receiving 5-FU infusions [5].

\section{Myocarditis and heart failure}

Myocarditis and $\mathrm{HF}$ are other manifestations of 5-FU cardiotoxicity. One case report described acute toxic myocarditis following a short infusion of 5-FU [41]. Subsequent coronary angiography demonstrated normal coronary arteries without evidence of coronary vasospasm. On left ventriculogram, global hypokinesis with an ejection fraction of $25 \%$ was noted. The patient was treated medically and left ventricular systolic function ultimately normalized. An additional case demonstrated global systolic dysfunction with 5-FU infusion and evidence of global myocarditis on diagnostic evaluation [42]. In a prospective study by Jensen et al. [43] significant NT-proBNP levels were noted in patients following 5-FU administration. In another case series, $3.5 \%$ of patients presented with acute decompensated HF during their first course of chemotherapy [13].

\section{Risk stratification}

Risk factors for cardiotoxicity with 5-FU include preexisting $\mathrm{CAD}$ or $\mathrm{HF}$, impaired renal clearance, prior symptoms with 5-FU administration, and longer infusion regimens $[3,14]$. Other predisposing factors for cardiotoxicity include prior chest irradiation and treatment with other chemotherapeutic agents with cardiac side effects [44]. Overall, patients with underlying $\mathrm{CAD}$ demonstrate rates of cardiotoxicity in the 5-15\% range [14, 45]. In addition to increasing the frequency of cardiotoxicity, the presence of preexisting heart disease increases the severity of cardiotoxic reactions to 5-FU [3]. Accordingly, a careful pre-administration cardiac evaluation is essential [46].

Despite the increased incidence of cardiotoxicity in patients with preexisting heart disease, the majority of cases occur in patients without any prior identified cardiac history and it is often difficult to predict which individual patient will be significantly adversely affected by the drug $[12,35]$. Thus, in conjunction with a thorough pre-treatment clinical assessment, frequent symptom, blood pressure, and ECG monitoring are essential components of any treatment protocol [46].

\section{Treatment and prophylaxis}

Following the recognition of acute cardiotoxicity, drug therapy should be ceased immediately, and treatment with nitrates or calcium channel blockers initiated (Table 4) [47]. In some studies, up to $70-90 \%$ of patients respond to such conservative therapy $[6,35]$. Others report response rates to these drugs of less than $50 \%[3,48]$.

Various preventive strategies have been pursued as well. Several studies have utilized oral capecitabine, an oral pro-drug of 5-FU, as an alternative agent to mitigate the adverse effects of 5-FU. Unfortunately, several reports still demonstrate drug-induced cardiotoxicity, albeit delayed until significant metabolite accumulation occurred $[1,25]$. Eskilsson et al. [48] attempted to prevent vasospasm-induced 5-FU cardiotoxicity via preadministration of verapamil without significant clinical effect. Another study by Salepci et al. [49] pursued prophylactic administration of ACE inhibitors for the prevention of 5-FU induced cardiomyopathy without effect. Another potentially promising preventive therapy is the pre-administration of a strong antioxidant like probucol, which has demonstrated 
Table 4. Management and re-challenging of patients with 5-fluorouracil (5-FU) cardiotoxicity [47].

\begin{tabular}{ll}
\hline Management of patients with 5-FU cardiotoxicity & Re-challenging patients with 5-FU cardiotoxicity* \\
\hline Stop administration of drug & Nitrates or calcium channel blockers 24 h before \\
Administration of nitrates or calcium channel blockers & re-challenge, during administration, and 24 h after \\
Monitor patients with cardiac enzymes; if elevated, & Continuous ECG monitoring during re-challenge \\
monitor an additional 72 h in an intensive care unit & Consider another chemotherapy (such as raltitrexed) \\
\hline
\end{tabular}

*If patients had acute myocardial infarction during 5-FU administration, then avoid re-challenge if possible

effectiveness in the lab, but whose efficacy has yet to be confirmed in human subjects [19]. Overall, most investigators suggest decrease in drug dosage as the most effective strategy [6, 48].

\section{Reintroduction of 5-FU}

Reintroducing 5-FU to patients with a history of cardiotoxicity following prior 5-FU administration is not currently advised. Repeated exposure to 5 -FU after documented cardiotoxicity carries a recurrence rate of $82-100 \%$ [17]. Moreover, a review of reexposure of 5-FU after cardiotoxicity revealed a death rate of $18 \%$ in patients who were reexposed to the drug [6]. A series by Clavel et al. [50] revealed that readministration of 5 -FU in a 28 patient cohort caused myocardial necrosis in 4 cases, and fatal cardiogenic shock in another 4 cases. If after careful consideration of the risks and benefits, rechallenge with 5-FU is deemed clinically appropriate, a reduction of dosage may be a viable option [51]. A prolonged course of pretreatment with nitrates and calcium channel blockers, continued during the drug infusion, has also been suggested [1]. Additionally, there is some evidence that in patients who develop cardiotoxicity to 5-FU, administration of another chemotherapy such as raltitrexed is an option [52]. Raltitrexed is a thymidylate synthase inhibitor that thus far does not show the cardiotoxic profile that 5 -FU seems to have, and may be an alternative in high risk patients [53]. Regardless, close monitoring in an inpatient setting is mandated for patients undergoing rechallenge with 5-FU following cardiotoxicity (Table 4). In our case, the patient ultimately resumed 5-FU therapy under close monitoring in an effort to achieve complete cure of his malignancy and experienced no further treatment complications.

\section{Conclusions}

With increased usage of 5-FU for the treatment of gastrointestinal malignancies, cardiotoxicities may be expected to be encountered more frequently in the future. A pre-chemotherapy history and physical is paramount and all patients should have a careful evaluation for cardiovascular risk factors as well as any subclinical CAD or cardiomyopathies which may be exacerbated by $5-\mathrm{FU}$ infusion. Although asymptomatic ECG changes, mild angina, and stable supraventricular arrhythmias predominate, dangerous complications such as MI, ventricular arrhythmias, decompensated HF, and death may also result from 5-FU administration. The etiology of 5-FU induced cardiotoxicity is probably multifactorial and may include coronary vasospasm, direct endothelial and myocardial injury, and accumulation of toxic metabolites. Pre-treatment with nitrates or calcium channel blockers may help to reduce the incidence of toxic effects although to date the only demonstrated effective strategies involve dose decreases or cessation of therapy. For those patients undergoing drug readministration following cardiotoxicity, therapy should take place in a carefully monitored setting. Overall, successful administration of 5-FU based therapies should involve a multidisciplinary approach to maximize clinical benefit and reduce potential harm.

\section{Conflict of interest: none declared}

\section{References}

1. Kosmas C, Kallistratos M, Kopterides P et al. Cardiotoxicity of fluoropyrimidines in different schedules of administration: A prospective study. J Cancer Res Clin Oncol, 2008; 134: 75-82.

2. Labianca R, Beretta G, Clerici M, Frascini P, Luporini G. Cardiac toxicity of 5-flurouracil: A study of 1083 patients. Tumori, 1982; 68: 505-510.

3. Jensen S, Sorensen J. Risk factors and prevention of cardiotoxicity induced by 5 -fluorouracil or capecitabine. Cancer Chemother Pharmacol, 2006; 58: 487-493.

4. Mosseri M, Fingert H, Varticovski L, Chokshi S, Isner J. In vitro evidence that myocardial ischemia resulting from 5 -fluorouracil chemotherapy is due to protein kinase c-mediated vasoconstriction of vascular smooth muscle. Cancer Res, 1993; 53: 3028-3033 .

5. Stewart T, Pavlakis N, Ward M. Cardiotoxicity with 5-fluorouracil and capecitabine: more than just vasospastic angina. Inter Med J, 2010; 40: 303-307.

6. Saif M, Shah M, Shah A. Fluoropyrimidine-associated cardiotoxicity: Revisited. Expert Opin Drug Saf, 2009; 8: 191-202. 
7. Sorrentino M, Truesdell A. 5-fluorouracil-induced coronary thrombosis: A case report and review of the literature. J Cardiol Cases, 2012: http://dx.doi.org/10.1016/j.jccase.2012.03.011.

8. Paoletti X, Oba K, Burzykowski et al. Benefit of adjuvant chemotherapy for resectable gastric cancer: a meta-analysis. JAMA, 2010; 303: 1729-1737.

9. Gianni L, Sessa C, Capri G, Grasselli G, Bioanchi G, Vitali G. Farmaci chemoterapici. In: Bonadonna G, Robustelli Della Cuna G, Valgusa $\mathrm{P}$ eds. Medicina oncological, $7^{\text {th }} \mathrm{Ed}$. Masson, Milano 2003: 583-676.

10. Anand A. Fluorouracil cardiotoxicity. Ann Pharmacother, 1994; 28: 374 .

11. Akhtar S, Salim K, Bano Z. Symptomatic cardiotoxicity with high dose 5-fluorouracil infusion: A prospective study. Oncology, 1993; 50: 441.

12. De Forni M, Malet-Martino M, Jaillais P et al. Cardiotoxicity of high-dose continuous infusion florouracil: A prospective clinical study. J Clin Oncol, 1992; 10: 1795-1801.

13. Meyer C, Calis K, Burke L, Walawander C, Grasela T. Symptomatic cardiotoxicity associated with 5-fluorouracil. Pharmacotherapy, 1997; 17: 729-736.

14. Meydan N, Kundak I, Yavuzsen T et al. Cardiotoxicity of de Gramont's regimen: incidence, clinical characteristics and long-term follow-up. Jpn J Clin Oncol, 2005; 35: 265-270.

15. Tsibiribi P, Descotes J, Lombard-Bohas C et al. Cardiotoxicity of 5-fluorouracil in 1350 patients with no prior history of heart disease. Bull Cancer, 2006; 93: 27-30.

16. Rezkalla S, Kloner R, Ensley J et al. Continuous ambulatory ECG monitoring during flurouracil therapy: A prospective study. J Clin Oncol, 1989; 7: 509-514.

17. Becker K, Erckenbrecht J, Haussinger D, Frieling T. Cardiotoxicity of the antiproliferative compound fluorouracil. Abstract. Drugs, 1999; 57: 475-484.

18. Gorgulu S, Celik S, Tezel T. A case of coronary spasm induced by 5-fluorouracil. Acta Cardiol, 2002; 57: 381-383.

19. Kinhult S, Albertsson M, Eskilsson J, Cwikiel M. Effects of probucol on endothelial damage by 5 -fluorouracil. Acta Oncologica, 2003; 42: 304-308.

20. Cwikiel M, Eskilsson J, Wieslander JB, Stjernquist U, Albertsson M. The appearance of endothelium in small arteries after treatment with 5 -fluorouracil. An electron microscopic study of late effects in rabbits. Scanning Microsc, 1996; 10: 805-818.

21. Luwaert R, Descamps O, Majois F, Chaudron J, Beauduin M. Coronary artery spasm induced by 5 -flurouracil. Eur Heart J, 1991; 12: 468-470.

22. Shoemaker L, Arora U, Rocha Lima C. 5-fluorouracil-induced coronary vasospasm. Cancer Control, 2004; 11: 46-49.

23. Sudhoff T, Enderle M, Pahlke M et al. 5-fluorouracil induces arterial vasocontractions. Ann Oncol, 2004; 15: 661-664.

24. Farina A, Malafronte C, Valsecchi M, Achilli F. Capecitabine-induced cardiotoxicity: When to suspect? How to manage? A case report. J Cardiovasc Med, 2009; 10: 722-726.

25. Senturk T, Kanat O, Evrensel T, Aydinlar A. Caecitabine-induced cardiotoxicity mimicking myocardial infarction. Neth Heart J, 2009; 17: 277-280.

26. Cwikiel M, Albertsson M, Zhang B et al. The influence of 5 -fluouracil on the endothelium in small arteries. A scanning and transmission electron microscopic study in rabbits. Scan Micros, 1995; 9: 561-576.

27. Kuzel T, Esyaraz B, Green D et al. Thrombogenicity of intravenous 5-fluorouracil alone or in combination with cisplatin. Cancer, 1990; 65: 885-889.

28. Cwikiel M, Eskilsson J, Albertsson M, Stavenow L. The influence of 5-fluorouracil and methotrexate on vascular endothelium. An experimental study using endothelial cells in the culture. Ann Oncol, 1996; 76: 731-737.

29. Tsibiribi P, Bui-Xuan C, Bui-Xuan B et al. Cardiac lesions induced by 5 -fluorouracil in the rabbit. Hum Exp Toxicol, 2006; 25: 305-309.

30. Kuropkat C, Griem K, Clark J, Rodriguez R, Hutchinson J, Taylor S. Severe cardiotoxicity during 5-flurorouracil chemotherapy: A case and literature support. Am J Clin Oncol, 1999; 22: 466.
31. Muneoka K, Shirai Y, Yokoyama N et al. 5-fluorouracil cardiotoxicity induced by alpha-fluoro-beta-alanine. Int J Clin Oncol, 2005; 10: 441-443.

32. Spasojevic I, Maksimovic V, Zakrzewska J, Bacic G. Effects of 5 -fluorouracil on erythrocytes in relation to its cardiotoxicity: Membrane structure and functioning. 2005; 45: 1680-1685.

33. Karabay C, Gecmen C, Aung S et al. Is 5-fluorouracil-induced vasospasm a Kounis syndrome? A diagnostic challenge. Perfusion, 2011; 26: 543-545.

34. Eskilsson J, Albertsson M, Mercke C. Adverse cardiac effects during induction chemotherapy treatment with cis-platin and 5-fluorouracil. Radiother Oncol, 1988; 13: 41-46.

35. Robben N, Pippas A, Moore J. The syndrome of 5-fluorouracil cardiotoxicity. Cancer, 1993; 71: 493-509.

36. Pottage A, Hold S, Ludgate S, Langlands A. Flurorouracil cardiotoxicity. Br Med J, 1978; 1: 547.

37. Wacker A, Lersch C, Scherpinski U, Reindl L, Seyfarth M. High incidence of angina pectoris in patients treated with 5-fluorouracil: A planned surveillance study with 102 patients. Oncology, 2003; 65: 108-112.

38. Abbott J, Curtis J, Murad K et al. Spontaneous coronary artery dissection in a woman receiving 5-fluorouracil: A case report. Angiology, 2003; 54: 721-724.

39. Talapatra K, Rajesh I, Rajesh B, Selvamani B, Subhashini J. Transient asymptomatic bradycardia in patients with infusional 5-fluroruracil. J Cancer Res Ther, 2007; 3: 169-171.

40. Oztop I, Gencer M, Okan T et al. Evaluation of cardiotoxicity of a combined bolus plus infusional 5-fluuorouracil/folinic acid treatment by echocardiography, plasma troponin I level, QT interval and dispersion in patients with gastrointenstinal system cancers. Jpn J Clin Oncol, 2004; 34: 262-268.

41. Calik A, Celiker E, Velibey Y, Cagdas M, Guzelburc O. Initial dose effect of 5-flurouracil: Rapidly improving severe, acute toxic myopericarditis. Am J Em Med, 2012; 30: 257.e1-257.e3.

42. Dalzell J, Samuel L. The spectrum of 5-fluorouracil cardiotoxicity. Anti-Cancer Drugs, 2009; 20: 79-80.

43. Jensen S, Hasbak P, Mortensen J, Sorensen J. Fluorouracil induced myocardial ischemia with increases of plasma brain natriuretic peptide and lactic acid but without dysfunction of left ventricle. J Clin Oncol, 2010; 28: 5280-5286.

44. Pai V, Nahata M. Cardiotoxicity of chemotherapeutic agents: Incidence, treatment and prevention. Drug Safety, 2000; 22: 263-302.

45. Schoeber C, Papageorgiou E, Harstrick A et al. Cardiotoxicity of 5 -fluorouracil in combination with folinic acid in patients with gastrointestinal cancer. Cancer, 1993; 72: 2242-2247.

46. Hrovatin E, Viel E, Lestuzzi C et al. Sever ventricular dysrhythmias and silent ischemia during infusion of the antimetabolite 5-fluorouracil and cis-platin. J Cardiovasc Med, 7: 637-640.

47. Cerny J, Hassan A, Smith C, Piperdi B. Coronary vasospasm with myocardial stunning in a patient with colon cancer receiving adjuvant chemotherapy with FOLFOX regimen. Clin Colo Cancer, 2009; 8: 55-58.

48. Eskilsson J, Albertsson M. Failure of preventing 5-fluorouracil cardiotoxicity by prophylactic treatment with verapamil. Acta Oncol, 1990; 29: 1001-1003.

49. Salepci T, Seker M, Uyarel H et al. 5-fluorouracil induced arterial vasoconstrictions but does not increase angiotensin II levels. Med Oncol, 2010; 27: 416-420.

50. Clavel M, Simeone P, Grivet B. Cardiac toxicity of 5-fluorouracil: Review of the literature, 5 new cases. Presse Med, 1988; 17: $1675-1678$.

51. Lestuzzi C, Crivellari D, Rigo F, Viel E, Meneguzzo N. Capacitabine cardiac toxicity presenting as effort angina: A case report. J Card Med, 2010; 11: 700-703.

52. Ng M, Cunniham D, Norman A. The frequency and pattern of cardiotoxicity observed with capecitabine used in conjunction with oxaliplatin in patients treated for advanced colorectal cancer. Eur J Cancer, 2005; 41: 1542-1546.

53. Kohne C, Thuss-Patience P, Friedrich $\mathrm{M}$ et al. Raltitrexed (Tomudex): An alternative drug for patients with colorectal cancer and 5-flurouracil associated cardiotoxicity. Br J Cancer, 1998; 77: 973-977. 